\title{
MicroRNA-122 protects against ischemic stroke by targeting Maf1
}

\author{
MENGMENG WANG $^{1}$, XIAOMAN LIU $^{1}$, YU WU ${ }^{2}$, YI WANG $^{1}$, JIAHUI CUI ${ }^{1}$, \\ JING SUN $^{3}$, YING BAI ${ }^{1}$ and MING-FEI LANG ${ }^{2}$
}

\author{
${ }^{1}$ Department of Neurology, Affiliated Xinhua Hospital of Dalian University, Dalian, Liaoning \\ $116021 ;{ }^{2}$ Medical College and ${ }^{3}$ College of Environmental and Chemical Engineering, Institute \\ of Microanalysis, Dalian University, Dalian, Liaoning 116622, P.R. China
}

Received March 7, 2020; Accepted September 11, 2020

DOI: $10.3892 / \mathrm{etm} .2021 .10048$

\begin{abstract}
The protection of brain tissue against damage and the reduction of infarct size is crucial for improving patient prognosis following ischemic stroke. Therefore, the present study aimed to investigate the regulatory effect of microRNA (miR)-122 and its target gene repressor of RNA polymerase III transcription MAF1 homolog (Maf1) on the infarct area in ischemic stroke. Reverse transcriptionquantitative PCR (RT-qPCR) was performed to determine miR-122 expression levels in an ischemic stroke [middle cerebral artery occlusion (MCAO)] mouse model. Nissl staining was conducted to measure the infarct area of the MCAO mouse model. Moreover, RT-qPCR was performed to investigate the relationship between the expression of Maf1 and miR-122 in the MCAO mouse model. Dual-luciferase reporter assay in vitro and miR-122 mimic or inhibitor treatment in vivo were conducted to verify that miR-122 targeted and inhibited Maf1 expression. The results suggested that miR-122 was upregulated in the brain tissue of MCAO model mice. miR-122 overexpression effectively reduced the size of the infarct area in comparison with a control and miR-122 knockdown in brain tissue resulted in the opposite effect. Moreover, Maf1 was confirmed to be a direct target of miR-122. The results of a dual-luciferase reporter assay indicated that miR-122 bound to the 3'-untranslated region of Maf1. Maf1 expression decreased after stroke model induction in comparison with that in sham animals, and Maf1 expression was negatively associated with the expression of miR-122. In addition, miR-122 knockdown increased
\end{abstract}

Correspondence to: Professor Ying Bai, Department of Neurology, Affiliated Xinhua Hospital of Dalian University, 156 Wansui Street, Dalian, Liaoning 116021, P.R. China

E-mail: 1029981825@qq.com

Professor Ming-Fei Lang, Medical College, Institute of Microanalysis, Dalian University, 10 Xuefu Avenue, Dalian, Liaoning 116622, P.R. China

E-mail: langmingfei@dlu.edu.cn

Key words: microRNA-122, repressor of RNA polymerase III transcription MAF1 homolog, ischemic stroke, infarct area
Maf1 expression levels, whereas miR-122 overexpression decreased Maf1 expression levels in comparison with a control. In conclusion, the results suggested that miR-122 improved the outcome of acute ischemic stroke by reducing the expression of Maf1.

\section{Introduction}

Stroke is the second largest cause of death worldwide $(1,2)$. The current treatment strategies for ischemic stroke are limited and early reperfusion is primarily established by intravenous thrombolysis or mechanical thrombectomy. However, there are no effective clinical drugs that improve patient prognosis following cerebral tissue infarction $(3,4)$.

MicroRNAs (miRNAs/miRs) are a class of non-coding RNAs that control the translation of the target protein through inhibition of the 3 '-untranslated region (UTR) of the target gene (5-7). It has been reported that $>33 \%$ of human genes are regulated by miRNAs (6). Previous studies have demonstrated that miRNAs are physiologically and pathologically involved in ischemic stroke $(7,8)$. Among them, miR-122 has been reported to be associated with ischemic stroke. miR-122 is encapsulated in exosomes and secreted into body fluids, where it is stable in serum and may serve as a promising biomarker for ischemic stroke $(9,10)$. miR-122 protects against amyloid- $\beta$-induced neuronal injury (11); however, the molecular mechanism underlying miR-122-mediated protection against loss of brain function is not completely understood.

It was initially determined that repressor of RNA polymerase III transcription MAF1 homolog (Maf1) was highly expressed in brain tissue with an abundance identified in the hippocampus and cortex (12). Subsequently TORC1 in the mTOR pathway was revealed to be involved in the phosphorylation of Maf1, which led to Maf1 inactivation (13). Since mTOR protects brain tissues from ischemic injuries, downregulation of Maf1 may have an inhibitory effect on the development of ischemic brain injuries $(14,15)$. In addition, $\mathrm{H}_{2} \mathrm{O}_{2}$ is produced by brain tissue after ischemic stroke, which can reduce cell function and further aggravate brain injury (16). $\mathrm{H}_{2} \mathrm{O}_{2}$ can serve as a messenger and promote the synthesis of the transcription factor Maf1 $(17,18)$. Therefore, it was hypothesized that Maf1 may serve an important role in the development and progression of ischemic stroke; however, the biological 
function of Maf1 and its relationship with ischemic stroke are not completely understood.

miR-122 may serve as a potential therapeutic target for ischemic stroke (19), whereas Maf1 may aggravate ischemic stroke, but the specific underlying mechanism is not completely understood (20-23). Therefore, it was hypothesized that miR-122 may be involved in the development of ischemic stroke by targeting Maf1. The present study aimed to investigate the relationship between Maf1 and miR-122 in the development and progression of ischemic stroke.

\section{Materials and methods}

Experimental animals. A total of 60 female C57BL/6 mice (age, 6-8 weeks; weight, 20-25 g) were purchased from the Experimental Animal Center of Dalian Medical University. The animals were housed at $23 \pm 2^{\circ} \mathrm{C}$ with $55 \pm 5 \%$ humidity under a 12-h light/dark cycle with free access to food and water. The present study was approved by the Animal Care Committee of the Xinhua Hospital affiliated to Dalian University and performed in accordance with the National Institutes of Health Guidelines (no. 85-23; revised 1996) for the Care and Use of Laboratory Animals (24).

Lateral ventricle injection. Mice were randomly divided into six groups ( $\mathrm{n}=6$ per group) for two experimental studies: The effect of miR-122 on infarct size after stroke (control, miR-122 mimic and miR-122 inhibitor groups) and the effect of miR-122 on Maf1 after stroke (control, miR-122 mimic and miR-122 inhibitor groups).

Firstly, $3.5 \mu \mathrm{l}$ miR-122 negative control (NC), mimic or inhibitor (cat. nos. B04002, B03001 and B02003; Shanghai GenePharma Co., Ltd.) was mixed with $3.5 \mu$ l RNAi-mate reagent (cat. no. G04002; Shanghai GenePharma Co., Ltd.) at room temperature for $15 \mathrm{~min}$ and stored for later use. After the mice were anesthetized with $400 \mathrm{mg} / \mathrm{kg} 4 \%$ chloral hydrate via intraperitoneal injection, the mixture (7 $\mu \mathrm{l})$ was injected into the lateral ventricle (over $20 \mathrm{~min}$ ) at the coordinate (bregma, $-2.5 \mathrm{~mm}$; dorsoventral, $1 \mathrm{~mm}$; lateral, $1.5 \mathrm{~mm}$ ). Subsequently, the wound was sutured and the mice were returned to the cage. At $24 \mathrm{~h}$ post-injection, the mouse middle cerebral artery occlusion (MCAO) model was established.

MCAO model. A total of 60 female C57BL/6 mice were selected for this model. The sham operated animals received the same operation as those in the experimental groups except for the coagulation of the blood vessels. The MCAO model (permanent coagulation of the distal middle cerebral artery) is highly reproducible and has a high success rate (the overall mortality is of $<5 \%$ ) (25). Moreover, the relative infarct volume in relation to brain size corresponds to the majority of human strokes (25). Mice were anesthetized with an intraperitoneal injection of $400 \mathrm{mg} / \mathrm{kg}$ chloral hydrate. After aseptic preparation of the surgical site, the skin between the ear and the eye was cut under a stereomicroscope (Shanghai Yuyan Instruments Co., Ltd.) using electrocoagulation forceps (Shanghai Yuyan Instruments Co., Ltd.). Once the temporal muscle was removed, a drill (Shanghai Yuyan Instruments Co., Ltd.) with a diameter of $2.5 \mathrm{~mm}$ was used to thin out the skull over the middle cerebral artery. The bone was carefully withdrawn to expose the middle cerebral artery, which was then coagulated with electrocoagulation forceps. Subsequently, the wound was sutured and the animal was maintained at $32^{\circ} \mathrm{C}$ for recovery (25). At $24 \mathrm{~h}$ post-model induction, 6 mice in each group were anesthetized with $400 \mathrm{mg} / \mathrm{kg}$ chloral hydrate and sacrificed by decapitation. Brain tissues were harvested and sectioned into 2-mm slices. Subsequently, the ischemic site was identified by incubation of the sections with $2 \%$ 2,3,5-triphenyl tetrazolium chloride (cat. no. T8170; Beijing Solarbio Science \& Technology Co., Ltd.) at $37^{\circ} \mathrm{C}$ for $15 \mathrm{~min}$ in the dark.

Coronal section. Brain tissues were fixed with $4 \%$ paraformaldehyde for $6 \mathrm{~h}$ at $4^{\circ} \mathrm{C}$, the surface liquid was removed by blotting and then the tissues were dehydrated with $15 \%$ sucrose solution overnight at $4^{\circ} \mathrm{C}$. Subsequently, the tissues were incubated with $30 \%$ sucrose solution overnight at $4^{\circ} \mathrm{C}$. Coronal sections were prepared into $30-\mu \mathrm{m}$ sections using a frozen microtome (Leica Microsystems $\mathrm{GmbH}$ ).

Nissl stain. Sections were washed twice with PBS for $5 \mathrm{sec}$ and stained with Nissl staining solution (cat. no. E607316; Sangon Biotech Co., Ltd.) for $15 \mathrm{~min}$ at $25^{\circ} \mathrm{C}$. Subsequently, the sections were washed twice with PBS for $5 \mathrm{sec}$ and then washed with $95 \%$ ethanol for $5 \mathrm{sec}$. Stained sections were examined under a Nikon Eclipse Ti inverted fluorescent microscope with a charge-coupled device camera at a magnification of $\mathrm{x} 100$. Microscopic images were analyzed with ImageJ (version 1.48; National Institutes of Health).

Prediction of miR-122 target gene. TargetScan (version 7.2; www.targetscan.org/vert_72), miRWalk (version 3; mirwalk. umm.uni-heidelberg.de) and miRDB (version 6.0; mirdb.org) gene prediction software were used to predict the target genes of miR-122. The intersection of the results was identified and used to investigate the association between target genes and ischemic stroke.

Reverse transcription-quantitative PCR (RT-qPCR). Total RNA was extracted from the cerebral cortex ( $n=6$ per group) using TRIzol ${ }^{\circledR}$ (cat. no. B610409; Sangon Biotech Co., Ltd.). Total RNA was reverse transcribed into cDNA using PrimeScriptTM RT reagent kit (cat. no. RR047A; Takara Biotechnology Co., Ltd.) at $37^{\circ} \mathrm{C}$ for $15 \mathrm{~min}$ and $85^{\circ} \mathrm{C}$ for 5 sec. Subsequently, qPCR was performed using SYBR Green Supermix (cat. no. RR820Q; Takara Biotechnology Co., Ltd.). The following thermocycling conditions were used for qPCR: Denaturation at $95^{\circ} \mathrm{C}$ for $30 \mathrm{sec}$; followed by 40 cycles of $95^{\circ} \mathrm{C}$ for $10 \mathrm{sec}$ and $60^{\circ} \mathrm{C}$ for $30 \mathrm{sec}$; and cooling to $4^{\circ} \mathrm{C}$. The following primers were used for qPCR: miR-122 (cat. no. ssD809230039; Sangon Biotech Co., Ltd.); Maf1 forward, 5'-GATTGCCACCCTCAATGAGTCC-3' and reverse, 5'-CTCCTCATCCACTGCATTCCAC-3' (Sangon Biotech Co., Ltd.); CEL-39 (cat. no. ssD1083145001; Sangon Biotech Co., Ltd.); and actin- $\beta$ (ACTB) forward, 5'-GTGCTA TGTTGCTCTAGACTTCG-3' and reverse, 5'-ATGCCACAG GATTCCATACC-3' (Sangon Biotech Co., Ltd.). miRNA and mRNA expression levels were analyzed using the $2^{-\Delta \Delta C q}$ 
Table I. Maf1 is predicted to be a target gene for miR-122.

RNA Predicted consequential pairing

mmu-miR-122 3' GUUUGUGGUAACAGUGUGAGGU mouse-Maf1 5' ...UCUUAUUGGGCCUGUA ФAФ४ФФA... rat-Maf1 5' ...CUUAUUGGGCCUGUACACUCCA... human-Maf1 5' ...UUCUACUGGGCCUGCACACUCCA...

Maf1,repressor of RNA polymerase III transcription MAF1 homolog.
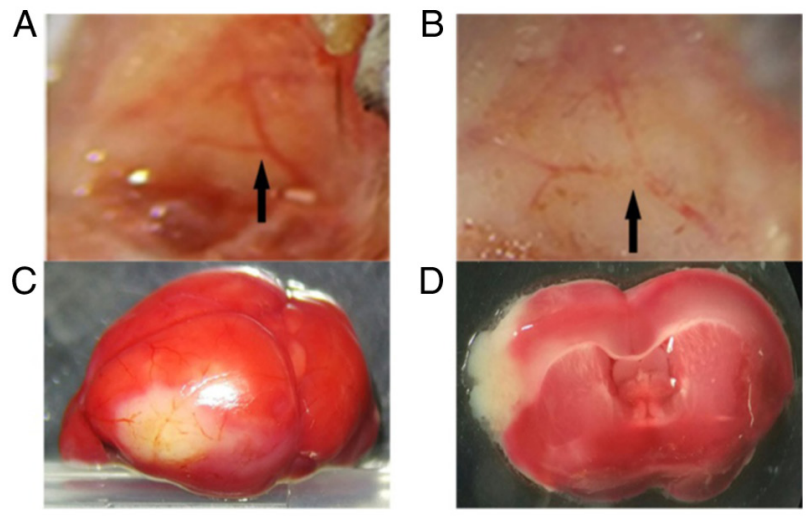

Figure 1. MCAO model establishment. (A) The arrow indicates the middle cerebral artery which was not coagulated. (B) The arrow indicates the middle cerebral artery which was coagulated using electrocoagulation forceps. (C) Representative image of TTC-stained brain surface after ischemic stroke modeling. Pale areas indicate ischemic focal points. (D) Representative image of TTC-stained coronary surface after MCAO. Pale areas indicate ischemic focal points. MCAO, middle cerebral artery occlusion; TTC, 2,3,5-triphenyl tetrazolium chloride.
A

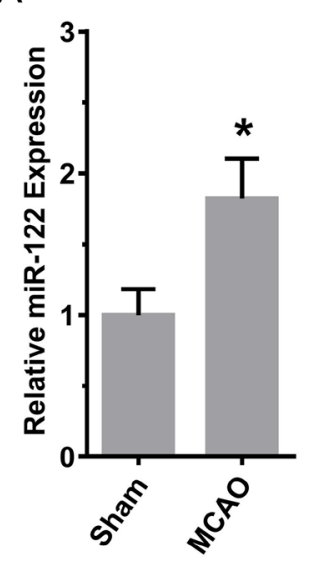

B
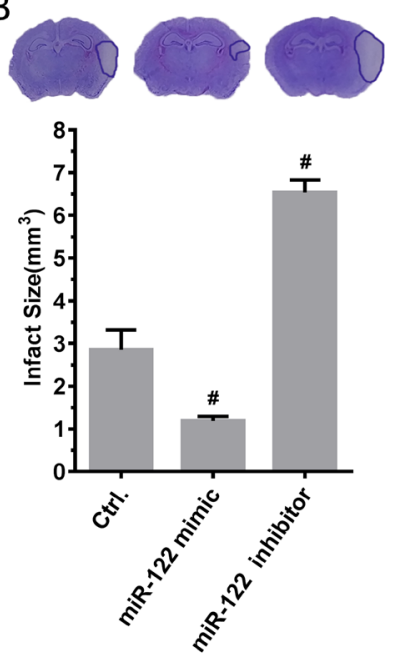

Figure 2. miR-122 overexpression in MCAO model rats effectively reduces the infarct area. (A) miR-122 expression in brain tissue. (B) Alterations to the infarct area after lateral ventricle injection of miR-122 NC, mimic or inhibitor. ${ }^{*} \mathrm{P}<0.05$ vs. sham; ${ }^{\#} \mathrm{P}<0.05$ vs. control. miR, microRNA; MCAO, middle cerebral artery occlusion; $\mathrm{NC}$, negative control.

method (26) and normalized to the internal reference genes CEL-39 and ACTB, respectively.
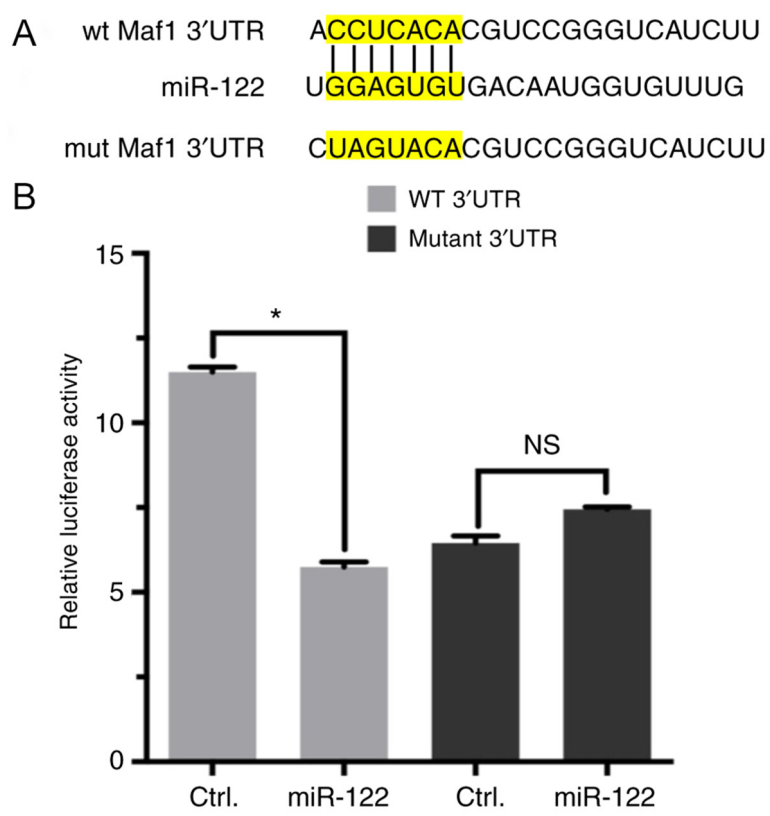

Figure 3. Maf1 is a direct target of miR-122. (A) The sequence of miR-122/Maf1-WT-3'UTR/Maf1-MUT-3'UTR. Yellow represents the miR-122 and Maf1-WT-3'UTR function sites and the Maf1-MUT-3'UTR mutation sites. (B) Luciferase activity of 293A cells following transfection with miRNA and vector for 48 h. ${ }^{*} \mathrm{P}<0.05$ vs. control. Ctrl, control; Maf1, repressor of RNA polymerase III transcription MAF1 homolog; miR, microRNA; WT, wild-type; UTR, untranslated region; MUT, mutant; NS, not significant.

Construction of vector. Maf1-3'UTR primers were designed by Sangon Biotech Co., Ltd. (forward, 5'-ATCCAGCTCTGA CCAATC-3' and reverse, 5'-CCAGGTTCCATCTAAGTC AC-3'). The PCR products were cloned into the XhoI and NotI sites of the siCheck vector (Invitrogen; Thermo Fisher Scientific, Inc.) to construct the Maf1-wild-type (WT)-3'UTR vector. The 3'UTR of Maf1 containing the predicted binding site of miR-122 was subjected to site-directed mutation (Sangon Biotech Co., Ltd.) to construct the Maf1-mutant (MUT)-3'UTR vector.

Dual-luciferasereporterassay.Cells(293A) weredivided into the following four groups: i) Maf1-WT-3'UTR + miR-122 NC (Maf1-WT-3'UTR + NC); ii) Maf1-WT-3'UTR + miR-122 mimic (Maf1-WT-3'UTR + miR-122); iii) Maf1-MUT-3'UTR vector + miR-122 NC (Maf1-MUT-3'UTR + NC); and iv) Maf1-MUT-3'UTR vector + miR-122 mimic (Maf1-MUT-3'UTR + miR-122). 293A cells (Dalian University Microanalysis Laboratory) were plated in 24-well plates at a density of $1 \times 10^{5}$ cells per well in DMEM (Thermo Fisher Scientific, Inc.) containing 10\% FBS (Thermo Fisher Scientific, Inc.). Cells were incubated at $37^{\circ} \mathrm{C}$ and $5 \% \mathrm{CO} 2$ and cultured to $90-95 \%$ confluence. Subsequently, cells were co-transfected with $50 \mathrm{nM}$ miRNA and $1 \mu \mathrm{g}$ vector using Lipofectamine ${ }^{\circledR} 2000$ (cat. no. 11668019; Thermo Fisher Scientific, Inc.). At 48 h post-transfection, luciferase activity was assessed using a dual-luciferase reporter assay kit (cat. no. RG027; Beyotime Institute of Biotechnology) and a fluorescence microplate reader at a wavelength of $560 \mathrm{~nm}$ for luciferin and $465 \mathrm{~nm}$ for renilla luciferase). 
A

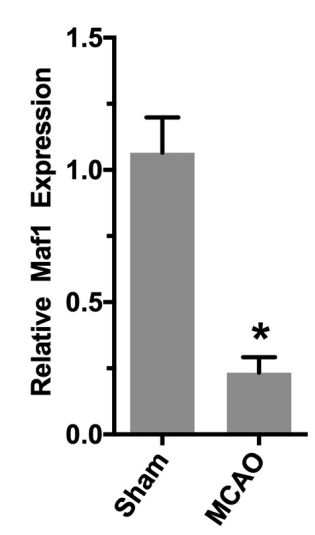

B

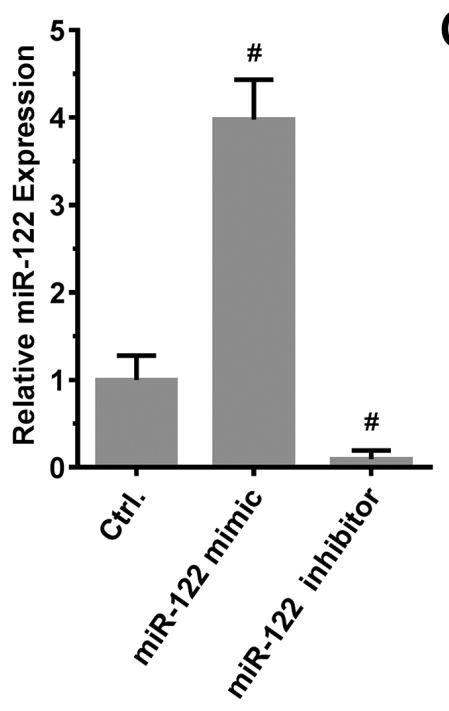

C

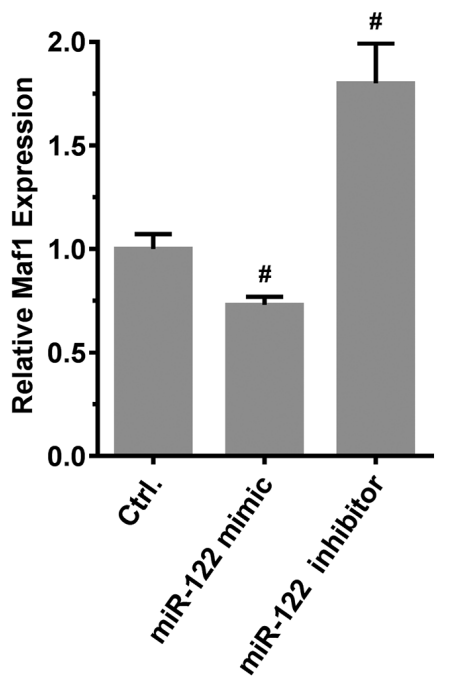

Figure 4. miR-122 inhibits the expression of Maf1. (A) Maf1 expression levels in the sham operation and MCAO groups. (B) miR-122 expression in the brain tissue after lateral ventricle injection of miR-122 NC, mimic or inhibitor. (C) Maf1 expression levels in the brain tissue following injection of miR-122 NC, mimic or inhibitor into the lateral ventricle. ${ }^{*} \mathrm{P}<0.05$ vs. sham; ${ }^{*} \mathrm{P}<0.05$ vs. control. miR, microRNA; Maf1, repressor of RNA polymerase III transcription MAF1 homolog; NC, negative control.

Firefly luciferase activity was normalized to Renilla luciferase activity.

Statistical analysis. Statistical analyses were conducted using SPSS software (version 22.0; IBM Corp.). The data were analyzed using unpaired Student's t-test or one-way ANOVA and Tukey's post-hoc test. Data are expressed as the mean \pm standard deviation. $\mathrm{P}<0.05$ was considered to indicate a statistically significant difference. All experiments were performed at least six times.

\section{Results}

Establishment of the MCAO model. As presented in Fig. 1A, the middle cerebral artery was exposed in a Y shape. After the middle cerebral artery was coagulated using electrocoagulation forceps, spontaneous recanalization was not observed (Fig. 1B). After MCAO establishment, the infarct brain area was white in color, whereas healthy brain tissues were stained red (Fig. 1C and D).

miR-122 decreases the infarct area. To investigate the relationship between miR-122 and ischemic stroke, the expression level of miR-122 was examined and the effect of miR-122 on the infarct area was evaluated. As presented in Fig. 2A, the expression of miR-122 (sham, 1.00 \pm 0.06 ; MCAO, 1.82 \pm 0.10 ; $\mathrm{P}<0.05 ; \mathrm{n}=6$ ) was significantly increased after MCAO induction in comparison with sham animals (Fig. 2A). Compared with MCAO model rats treated with control miRNA, the size of the area of infarction was significantly reduced in MCAO model rats that were injected with miR-122 mimic in the lateral ventricle in comparison with control animals. By contrast, the area of infarction increased significantly in comparison with the control following injection of miR-122 inhibitor in the lateral ventricle (control, 2.85 \pm 0.27 ; mimic, $1.19 \pm 0.05$; inhibitor $6.53 \pm 0.17$; mimic vs. control and inhibitor vs. control, $\mathrm{P}<0.05$; $\mathrm{n}=6$; Fig. $2 \mathrm{~B})$.
Predicted target gene of $m i R-122$. To reduce the false positive probability of the target gene prediction, gene prediction was performed using the intersection of the prediction results obtained from the three different types of gene prediction software: TargetScan, miRWalk and miRtaget2. Maf1 was identified as a target gene of miR-122 in humans, mice and rats (Table I).

Mafl is a direct target of $m i R-122$. After sequence analysis, it was observed that the 3'UTR of Maf1 was a putative target of miR-122 (Fig. 3A). As the 3'UTR of Maf1 mRNA was predicted to contain a target region of miR-122, a dual-luciferase reporter assay was conducted to investigate whether miR-122 directly targeted Maf1. 293a cells were transfected with Maf1-WT-3'UTR + NC as a negative control. Compared with the negative controls, co-transfection with miR-122 reduced the luciferase activity of Maf1-WT-3'UTR vector (control, 11.5 \pm 0.10 ; miR-122, 5.8 $\pm 0.00 ; \mathrm{P}<0.01$; Fig. 3B). However, there was no significant alteration to the luciferase activity of Maf1-MUT-3'UTR vector following co-transfection with miR-122 mimics compared with co-transfection with miR-122 mimics negative control (control, 6.5 \pm 0.15 ; miR-122, $7.5 \pm 0.05$; P>0.05; Fig. 3B).

miR-122 inhibits Maf1 expression. Subsequently, whether miR-122 inhibited Maf1 expression in vivo was investigated. The results demonstrated that compared with the sham group, the expression of Maf1 in the MCAO group was significantly reduced (sham, 1.00 $\pm 0.04 ; \mathrm{MCAO}, 0.21 \pm 0.04 ; \mathrm{P}<0.01 ; \mathrm{n}=6$; Fig. 4A). Furthermore, RT-qPCR results suggested that the expression of Maf1 was significantly reduced after injection of miR-122 mimic in the lateral ventricle. By contrast, the expression of Maf1 increased significantly after the injection of miR-122 inhibitor in the lateral ventricle. The expression levels of miR-122 were as follows: $1.00 \pm 0.16,3.98 \pm 0.26$ and $0.10 \pm 0.04$ (mimic vs. control and inhibitor vs. control, $\mathrm{P}<0.05$; n=6; Fig. 4B). Maf1 expression levels were as follows: 
$1.00 \pm 0.03,0.73 \pm 0.02$ and $1.80 \pm 0.09$ (mimic vs. control and inhibitor vs. control, $\mathrm{P}<0.05 ; \mathrm{n}=6$; Fig. 4C).

\section{Discussion}

It has been reported that there is a close relationship between miRNA and ischemic stroke $(1,27,28)$, with the expression of $\sim 20 \%$ miRNAs changing during the development and progression of ischemic stroke (29). By regulating the translation process of mRNA, miRNAs can affect protein expression, which subsequently alters biological functions such as axon regeneration, angiogenesis and inflammatory responses (30-33). Several studies have indicated that miR-122 can maintain the caliber and tube shape of blood vessels and reduce neuronal death in an ischemic stroke model $(19,34)$. In addition, miR-122 may serve a pivotal immunomodulatory role in hepatocytes via miR-122-RTKs/STAT3-IRF1-IFNs regulatory circuitry or target TLR4 $(35,36)$. Nerve cells, blood vessels and immunity are critical to the prognosis of infarct area in ischemic stroke $(37,38)$. Therefore, the present study investigated the effect of miR-122 on the outcome of ischemic stroke. Compared with the level in the sham group, the expression of miR-122 increased significantly after MCAO. In addition, increased miR-122 expression effectively reduced the infarct area. By contrast, inhibition of miR-122 expression in brain tissue significantly increased the infarct area, which was consistent with previous studies $(19,39)$. Therefore, the results indicated that miR-122 may exert a protective effect against ischemic stroke by reducing the infarct area.

miRNAs function by regulating the expression of downstream target genes (1); therefore, studying the downstream target genes of miR-122 during the development and progression of ischemic stroke may be important.

Previous studies have indicated that the Maf1 gene is associated with oxygen free radicals, such as $\mathrm{H}_{2} \mathrm{O}_{2}$, and the mTOR signaling pathway induced by ischemia $(17-18,20)$. It has also been speculated that the Maf1 gene is associated with ischemic stroke-induced cell death, which further exacerbates brain damage (21-23). The present study demonstrated that the 3'UTR of Maf1 mRNA was a binding site for miR-122 using gene prediction software. Therefore, Maf1 was investigated as a target gene of miR-122. A dual-luciferase reporter assay was performed, which verified that miR-122 bound to the 3'UTR of Maf1. In addition, after MCAO, the expression of miR-122 was increased in comparison with that in a sham model, whereas the expression of Maf1 was reduced, indicating that miR-122 may be negatively associated with Maf1 expression. Therefore, it was hypothesized that the expression of Maf1 may be inhibited by miR-122 after MCAO. Increased miR-122 expression inhibited Maf1 expression and decreased miR-122 expression increased Maf1 expression in vivo. In summary, the present study indicated that miR-122 inhibited Maf1 expression after MCAO. The results of the present study may be beneficial in evaluating the relationship between Maf1 and ischemic stroke and also indicated that miR-122 may be able to improve the outcome of ischemic stroke by regulation of the Maf1 gene. However, the specific role and regulatory mechanism underlying Maf1 and miR-122 activity during the development of ischemic stroke are not completely understood and require further investigation. In contrast to other studies that used male mice, only female mice were used in the present study. Therefore, potential differences in findings in experiments using male mice cannot be ruled out. More studies with male mice may further clarify whether miR-122 promotes the outcome of acute ischemic stroke by reducing the expression of Maf1.

\section{Acknowledgements}

Not applicable.

\section{Funding}

The present study was funded by the Joint Foundation of Natural Science Foundation of Liaoning Province and Shenyang National Laboratory for Materials Science (grant no. 2019JH3/30100006), the National Natural Science Foundation of China (grant no. 21505013), the Liaoning BaiQianWan Talents Program [grant no. (2019)45] and Dalian Science and Technology Innovation Funds (grant no. 2018J13SN087).

\section{Availability of data and materials}

The datasets used and/or analyzed during the current study are available from the corresponding author on reasonable request.

\section{Authors' contributions}

MW, YB and ML conceived and designed the study, and MW performed the majority of the experiments. MW, XL and YW constructed the MCAO model. YW, JC and JS cultured the cells. MW wrote the manuscript. All authors read and approved the manuscript and agree to be accountable for all aspects of the research.

\section{Ethics approval and consent to participate}

The present study was approved by the Animal Care Committee of the Xinhua Hospital affiliated to Dalian University and performed in accordance with the National Institutes of Health Guidelines for the Care and Use of Laboratory Animals.

\section{Patient consent for publication}

Not applicable.

\section{Competing interests}

The authors declare that they have no competing interests.

\section{References}

1. Zhou J, Chen L, Chen B, Huang S, Zeng C, Wu H, Chen C and Long F: Increased serum exosomal miR-134 expression in the acute ischemic stroke patients. BMC Neurol 18: 198, 2018.

2. Zheng M, Wang X, Yang J, Ma S, Wei Y and Liu S: Changes of complement and oxidative stress parameters in patients with acute cerebral infarction or cerebral hemorrhage and the clinical significance. Exp Ther Med 19: 703-709, 2020. 
3. Catanese L, Tarsia J and Fisher M: Acute ischemic stroke therapy overview. Circ Res 120: 541-558, 2017.

4. Bai Y, Zhang Y, Han B, Yang L, Chen X, Huang R, Wu F, Chao J, Liu P, Hu G, et al: Circular RNA DLGAP4 ameliorates ischemic stroke outcomes by targeting miR-143 to regulate endothelial-mesenchymal transition associated with blood-brain barrier integrity. J Neurosci 38: 32-50, 2018.

5. Wang SW, Liu Z and Shi ZS: Non-Coding RNA in acute ischemic stroke: Mechanisms, biomarkers and therapeutic targets. Cell Transplant 27: 1763-1777, 2018.

6. Qi Z, Cai S, Cai J, Chen L, Yao Y, Chen L and Mao Y: miR-491 regulates glioma cells proliferation by targeting TRIM28 in vitro. BMC Neurol 16: 248, 2016.

7. Qi R, Liu H, Liu C, Xu Y and Liu C: Expression and short-term prognostic value of miR-126 and miR-182 in patients with acute stroke. Exp Ther Med 19: 527-534, 2019.

8. Deng Y, Chen D, Gao F, Lv H, Zhang G, Sun X, Liu L, Mo D, Ma N, Song L, et al: Exosomes derived from microRNA-138-5p-overexpressing bone marrow-derived mesenchymal stem cells confer neuroprotection to astrocytes following ischemic stroke via inhibition of LCN2. J Biol Eng 13: 71, 2019.

9. Stanzione R, Bianchi F, Cotugno M, Marchitti S, Forte M, Busceti C, Ryskalin L, Fornai F, Volpe M and Rubattu S: A decrease of brain MicroRNA-122 level is an early marker of cerebrovascular disease in the stroke-prone spontaneously hypertensive rat. Oxid Med Cell Longev 2017: 1206420, 2017.

10. Li DB, Liu JL, Wang W, Luo XM, Zhou X, Li JP, Cao XL, Long XH, Chen JG and Qin C: Plasma exosomal miRNA-122-5p and miR-300-3p as potential markers for transient ischaemic attack in rats. Front Aging Neurosci 10: 24, 2018.

11. Gu R, Wang L, Tang M, Li SR, Liu R and Hu X: LncRNA Rpph1 protects amyloid- $\beta$ induced neuronal injury in SK-N-SH cells via miR-122/Wnt1 axis. Int J Neurosci 130: 443-453, 2020.

12. Smith KR, Oliver PL, Lumb MJ, Arancibia-Carcamo IL, Revilla-Sanchez R, Brandon NJ, Moss SJ and Kittler JT: Identification and characterisation of a Maf1/Macoco protein complex that interacts with GABAA receptors in neurons. Mo Cell Neurosci 44: 330-341, 2010.

13. Shetty M, Noguchi C, Wilson S, Martinez E, Shiozaki K, Sell C, Mell JC and Noguchi E: Maf1-dependent transcriptional regulation of tRNAs prevents genomic instability and is associated with extended lifespan. Aging Cell 19: e13068, 2020.

14. Yamada D, Kawabe K, Tosa I, Tsukamoto S, Nakazato R, Kou M, Fujikawa K, Nakamura S, Ono M, Oohashi T, et al: Inhibition of the glutamine transporter SNAT1 confers neuroprotection in mice by modulating the mTOR-autophagy system. Commun Biol 2: 346, 2019.

15. Ma HX, Hou F, Chen AL, Li TT, Zhu YF and Zhao QP $\mathrm{Mu}$-Xiang-You-Fang protects $\mathrm{PC} 12$ cells against OGD/R-induced autophagy via the AMPK/mTOR signaling pathway. J Ethnopharmacol 252: 112583, 2020.

16. Imai T, Iwata $S$, Miyo D, Nakamura S, Shimazawa $M$ and Hara H: A novel free radical scavenger, NSP-116, ameliorated the brain injury in both ischemic and hemorrhagic stroke models. J Pharmacol Sci 141: 119-126, 2019

17. Gunawardena D, Raju R and Münch G: Hydrogen peroxide mediates pro-inflammatory cell-to-cell signaling: A new therapeutic target for inflammation. Neural Regen Res 14: 1430-1437, 2019.

18. Sies H: Hydrogen peroxide as a central redox signaling molecule in physiological oxidative stress: Oxidative eustress. Redox Biol 11: 613-619, 2017

19. Liu da Z, Jickling GC, Ander BP, Hull H, Zhan X, Cox C, Shroff N, Dykstra-Aiello C, Stamova B and Sharp FR: Elevating microRNA-122 in blood improves outcomes after temporary middle cerebral artery occlusion in rats. J Cereb Blood Flow Metab 36: 1374-1383, 2016.

20. Tsang CK, Chen M, Cheng X, Qi Y, Chen Y, Das I, Li X, Vallat B, Fu LW, Qian CN, et al: SOD1 phosphorylation by mTORC1 couples nutrient sensing and redox regulation. Mol Cell 70 502-515, 2018

21. Liu BW, Luo C, Zheng ZG, Xia ZY, Zhang Q, Ke C, Liu R and Zhao YH: Shengui sansheng san extraction is an angiogenic switch via regulations of AKT/mTOR, ERK1/2 and Notch 1 signal pathways after ischemic stroke. Phytomedicine 44: 20-31, 2018.
22. Hou YY, Wang K, Wan WJ, Cheng Y, Pu X and Ye XF: Resveratrol provides neuroprotection by regulating the JAK2/STAT3/PI3K/AKT/mTOR pathway after stroke in rats. Genes Dis 5: 245-255, 2018.

23. Zhang SS, Li XX, Wang HY and Zheng XFS: Beyond regulation of pol III: Role of MAF1 in growth, metabolism, aging and cancer. Biochim Biophys Acta Gene Regul Mech 1861: 338-343, 2018.

24. National Research Council (US) Institute for Laboratory Animal Research: Guide for the Care and Use of Laboratory Animals. National Academies Press (US), Washington, DC, 1996.

25. Llovera G, Roth S, Plesnila N, Veltkamp R and Liesz A: Modeling stroke in mice: Permanent coagulation of the distal middle cerebral artery. J Vis Exp 31: e51729, 2014.

26. Livak KJ and Schmittgen TD: Analysis of relative gene expression data using real-time quantitative PCR and the 2(-Delta C(T)) method. Methods 25: 402-408, 2001.

27. Cheng X, Kan P, Ma Z, Wang Y, Song W, Huang C and Zhang B Exploring the potential value of miR-148b-3p, miR-151b and miR-27b-3p as biomarkers in acute ischemic stroke. Biosci Rep 38: BSR20181033, 2018.

28. Si W, Ye S, Ren Z, Liu X, Wu Z, Li Y, Zhou J, Zhang S, Li Y, Deng $R$ and Chen D: miR-335 promotes stress granule formation to inhibit apoptosis by targeting ROCK2 in acute ischemic stroke. Int J Mol Med 43: 1452-1466, 2019.

29. Liu W, Chen X and Zhang Y: Effects of microRNA-21 and microRNA-24 inhibitors on neuronal apoptosis in ischemic stroke. Am J Transl Res 8: 3179-3187, 2016.

30. Shi FP, Wang XH, Zhang HX, Shang MM, Liu XX, Sun HM and Song YP: MiR-103 regulates the angiogenesis of ischemic stroke rats by targeting vascular endothelial growth factor (VEGF). Iran J Basic Med Sci 21: 318-324, 2018.

31. Meng P, Zhu Q, Yang H, Liu D, Lin X, Liu J, Fan J, Liu X, Su W, Liu L, et al: Leonurine promotes neurite outgrowth and neurotrophic activity by modulating the GR/SGK1 signaling pathway in cultured PC12 cells. Neuroreport 30: 247-254, 2019.

32. Cheng J, Tang JC, Pan MX, Chen SF, Zhao D, Zhang Y, Liao HB Zhuang Y, Lei RX, Wang S, et al: 1-lysine confers neuroprotection by suppressing inflammatory response via microRNA-575/PTEN signaling after mouse intracerebral hemorrhage injury. Exp Neurol 327: 113214, 2020

33. Du K, Zhao C, Wang L, Wang Y, Zhang KZ, Shen XY, Sun HX, Gao W and Lu X: MiR-191 inhibit angiogenesis after acute ischemic stroke targeting VEZF1. Aging (Albany NY) 11: 2762-2786, 2019

34. Guo D, Ma J, Li T and Yan L: Up-regulation of miR-122 protects against neuronal cell death in ischemic stroke through the heat shock protein 70 -dependent $\mathrm{NF}-\kappa \mathrm{B}$ pathway by targeting FOXO3. Exp Cell Res 369: 34-42, 2018

35. Shi L, Zheng X, Fan Y, Yang X, Li A and Qian J: The contribution of miR-122 to the innate immunity by regulating toll-like receptor 4 in hepatoma cells. BMC Gastroenterol 19: 130, 2019.

36. Xu H, Xu SJ, Xie SJ, Zhang Y, Yang JH, Zhang WQ, Zheng MN, Zhou $\mathrm{H}$ and $\mathrm{Qu} \mathrm{LH}$ : MicroRNA-122 supports robust innate immunity in hepatocytes by targeting the RTKs/STAT3 signaling pathway. Elife 8: e41159, 2019.

37. Hatakeyama M, Ninomiya I and Kanazawa M: Angiogenesis and neuronal remodeling after ischemic stroke. Neural Regen Res 15: $16-19,2020$

38. Jayaraj RL, Azimullah S, Beiram R, Jalal FY and Rosenberg GA: Neuroinflammation: Friend and foe for ischemic stroke. J Neuroinflammation 16: 142, 2019.

39. Lv B, Cheng X, Sharp FR, Ander BP and Liu DZ: MicroRNA-122 mimic improves stroke outcomes and indirectly inhibits $\mathrm{NOS}_{2}$ after middle cerebral artery occlusion in rats. Front Neurosci 12: 767, 2018. 